\title{
Estado actual de la integración de tecnologías digitales en la enseñanza de idiomas en la Escuela de Lenguas Modernas de la Universidad de Costa Rica
}

\author{
Patricia Escalante Arauz \\ Escuela de Lenguas Modenas
} Universidad de Costa Rica

\begin{abstract}
Resumen
Debido al gran impacto que han tenido las tecnologías digitales en la sociedad del siglo XXI, y la importancia que tiene para los profesionales de todos los ámbitos integrarlas en su vida diaria y además dominar otros idiomas, surge la inquietud de conocer cómo se está haciendo esa integración en los cursos de la Escuela de Lenguas Modernas. Los docentes participaron en este estudio por medio de un cuestionario y un grupo focal para conocer su posición y experiencia con respecto a la integración de tecnología en el currículo. Además, los estudiantes también participaron respondiendo a un cuestionario relacionado con la forma en que ellos las integran no sólo en su vida diaria sino también en su quehacer académico.
\end{abstract}

Palabras claves: tecnologías digitales, capacitación docente, integración de tecnologías, aprendizaje de lenguas extranjeras, TIC

\begin{abstract}
The great impact digital technologies have had in the $21^{\text {st }}$ century society and the importance for professionals to integrate them in their daily lives, plus the need they have to speak other languages, led to the concern of knowing how that integration is taking place in the courses offered by the School of Modern Languages. The faculty participated in this study through a questionnaire and a focus group in order to know their positions and experiences in relation to integrating technology into the curriculum. The students also participated through a questionnaire related to the way they integrate them not only in their daily but also in their academic lives.
\end{abstract}

Key:words: digital technologies, teacher training, technology integration, foreign language learning, ICT 


\section{Antecedentes}

$\mathrm{E}$ n las últimas décadas, las tecnologías de la comunicación y la información (TIC) han tenido una gran presencia y se han propagado en todas las áreas del desarrollo humano, en especial en la del aprendizaje. Específicamente en el campo del aprendizaje de idiomas, las TIC también han provocado grandes expectativas por su potencial para mejorar las destrezas comunicativas. De acuerdo con un estudio realizado por el Centro de Estudios Avanzados del Lenguaje (CASL, por sus siglas en Inglés) de la Universidad de Maryland, Frank et al. (2008) encontraron que, cuando los docentes utilizan las herramientas tecnológicas en la enseñanza de lenguas extranjeras, lo hacen básicamente de cuatro formas:

- Herramientas para el uso en la clase: sistemas de manejo de cursos, pizarras inteligentes y herramientas para la evaluación de los aprendizajes.

- Herramientas de estudio individual: diccionarios electrónicos y programas para mejorar tanto la gramática como la pronunciación, entre otros.

- Herramientas para la interacción social: blogs, wikis, chats y mundos virtuales.

- Dispositivos portátiles con acceso a Internet: como tabletas y laptops para la búsqueda de información y aprendizaje ubicuo.

Además, el estudio citado concluyó que la integración de la tecnología tiene el potencial de mejorar cinco aspectos de la enseñanza y el aprendizaje de idiomas:
- Organización: La tecnología permite a docentes y estudiantes organizar el aprendizaje y la enseñanza fuera del aula; y a los últimos, reflexionar y tomar control de su propio aprendizaje.

- Input: La tecnología puede ampliar el acceso, de manera personalizada, a una mayor gama de recursos en el idioma meta.

- Output e interacción: La tecnología da a los estudiantes oportunidades de crear sus propias interacciones y producciones de manera sincrónica o asincrónica con hablantes nativos o con conocimientos más avanzados del idioma meta.

- Realimentación: Ofrece mayores oportunidades para dar y recibir realimentación individualizada y a tiempo.

- Colaboración: Permite el aprendizaje social y colaborativo de manera sincrónica y asincrónica dentro del aula y fuera de ella.

Ante tanto potencial de todos estos recursos tecnológicos y los desafíos de la sociedad actual, esta investigación se propone conocer cuánta integración de tecnología se da o no en los cursos ofrecidos por la Escuela de Lenguas Modernas. Para el contexto de este estudio en particular, el uso de tecnologías de la información y la comunicación (TIC) se refiere sobre todo a programas y aplicaciones computacionales, además de la Internet, que se usan para mejorar el aprendizaje de idiomas.

Las preguntas de base para esta investigación son las siguientes:

1. ¿Cuál es el statu quo del uso de las TIC que hace el profesorado?

2. ¿Con qué objetivo se utilizan y cómo? 
3. ¿Cuáles factores institucionales o de infraestructura influyen en su integración o falta de esta?

4. ¿Cuál es la visión tanto de los docentes como de los estudiantes en cuanto al uso de la tecnología para mejorar el aprendizaje?

\section{Marco teórico}

Es importante delinear una perspectiva teórica como base para el estudio sobre la integración de tecnologías digitales en el aprendizaje de idiomas. Para empezar, es preciso revisar algunas de las teorías relacionadas con la adquisición de una segunda lengua (SLA, por sus siglas en inglés). Varios investigadores en el tema (Pica, 1998; Ellis, 1997; Nunan, 1987; Krashen, 1981) apoyan una posición interaccionista y enfatizan los efectos del ambiente lingüístico externo y las diferencias individuales de aprendizaje sobre la adquisición de idiomas. Se propone que los principios del input comprensible, la negociación de significados y la atención tanto a la forma como al significado tienen un impacto en el progreso del interlenguaje del aprendiz.

El marco conceptual de la presente investigación refleja tanto las teorías interaccionistas como las perspectivas socioculturales en la adquisición de una segunda lengua. De acuerdo con la teoría del input de Krashen (1981), la cantidad de input comprensible (mensajes que los estudiantes puedan comprender) determina en gran medida la adquisición de una segunda lengua; esos mensajes, a su vez, deben ser de un nivel más allá de la competencia lingüística de los estudiantes. Esta teoría del andamiaje de Krashen se co- noce como $i+1$, de manera similar a la teoría de Vygotsky de la zona de desarrollo próximo (1962).

Es importante destacar que mientras Krashen se centra en la importancia del input comprensible para adquirir una segunda lengua, otros investigadores toman una posición interaccionista un poco diferente, al reconocer el papel de una comunicación en dos vías. Según Pica (1998), la interacción conversacional facilita la adquisición de una segunda lengua bajo ciertas circunstancias; por ejemplo, cuando los estudiantes tienen la oportunidad de involucrarse en actividades significativas se ven forzados a negociar significados, a expresar y clarificar sus intenciones, pensamientos y opiniones con el fin de lograr un mutuo entendimiento. Esto es especialmente cierto cuando los estudiantes trabajan juntos para alcanzar una meta en común.

Por otro lado, en su hipótesis de output comprensible (producción oral), Swain (1995) afirma que este tiene una gran importancia y aporta cuatro funciones esenciales para el estudiante: 1) mejorar la fluidez, 2) crear conciencia de brechas en el conocimiento de la lengua, 3) ofrecer oportunidades al estudiante para experimentar con estructuras y formas lingüísticas y 4) dar oportunidad de recibir realimentación de otros acerca del uso de la lengua. Es decir, el output comprensible ayuda a los estudiantes a expresarse significativamente mientras superan retos lingüísticos (Chapelle, 1998).

En resumen, los interaccionistas plantean la importancia de la interacción construida con base en intercambios de input y de output comprensibles, los cuales a su vez realzan la 
negociación de significados y el uso de estructuras gramaticales de apoyo.

Todas estas teorías dan sustento a la inquietud planteada por muchos docentes sobre por qué integrar las tecnologías en el aprendizaje de idiomas, y si realmente esa integración mejora el desempeño del estudiante. Una rama de la lingüística aplicada, llamada Computer Assisted Language Learning (CALL, por sus siglas en inglés), investiga desde los años sesentas la evolución del uso de tecnología en la enseñanza de idiomas (Levy, 1997).

Para el presente estudio, es importante revisar la literatura sobre prácticas de enseñanza e investigación relacionadas con los usos de las TIC en la enseñanza de idiomas. Tanto Egbert (2005) como Hanson-Smith y Rilling (2006) caracterizaron el CALL como ambientes de aprendizaje y enseñanza óptimos, realzados por la tecnología, es decir, lenguaje y escenarios de contenido en los cuales se usa la tecnología de la manera más efectiva para apoyar el aprendizaje. Como lo exponen Warschauer (1997) y Healey (1998), la forma de hacer la integración de las TIC está relacionada con la evolución de los enfoques pedagógicos empleados en la enseñanza de idiomas.

En los años setentas y ochentas, se le consideraba un enfoque comunicativo (CALL comunicativo), pues respondía a las teorías cognitivas que ven el aprendizaje como un proceso de descubrimiento, expresión y desarrollo. Por lo tanto, el centro del proceso educativo no estaba en la máquina sino en lo que los estudiantes podían hacer con esta mientras trabajaban entre ellos. En los años noventas, se desarrolló una nueva perspectiva, una visión sociocognitiva con mayor énfasis en el uso del idioma en contextos sociales auténticos y con la integración de las diferentes destrezas comunicativas, por lo cual los investigadores lo denominaron CALL integrador. Hubbard (2005) agrega que este campo ha evolucionado hacia un CALL inteligente (ICALL), el cual consiste en la integración de los siguientes elementos:

- Equipos móviles (laptops, tabletas, teléfonos celulares).

- La Web 2.0, con herramientas tecnológicas para la colaboración, como las redes sociales, publicación de textos como blogs y wikis y publicación de videos.

- Aprendizaje de idiomas móvil, el cual ofrece a los estudiantes la oportunidad de una conexión ubicua con aplicaciones y materiales en línea a los que pueden acceder en cualquier momento y desde cualquier lugar.

- Mundos virtuales, en donde los estudiantes pueden explorar, crear e interactuar con otros, hablantes nativos o estudiantes como ellos, alrededor del mundo.

Según lo afirman los seguidores del ICALL, al hacer una integración intencionada de las TIC en la enseñanza de idiomas (Hubbard, 2005; Warschauer, 1997), se va más allá del uso instrumental de herramientas de ofimática. Así, los diferentes programas y aplicaciones usados con propósitos pedagógicos son el medio para que los estudiantes logren construir su propio conocimiento y, a la vez, propiciar un aprendizaje colaborativo y autónomo, de manera que no dependan tanto del profesor ni de la clase presencial.

Como lo planteara Swain (1995), el output comprensible ayuda a los 
estudiantes a expresarse significativamente mientras superan retos lingüísticos. Con el uso de Internet y en especial de las redes sociales y textos de blogs y wikis, por ejemplo, los estudiantes tienen acceso a hablantes nativos y estudiantes avanzados del idioma meta en cualquier momento y en cualquier lugar. Esto les abre una ventana de posibilidades de aprendizaje colaborativo y de realimentación sobre su producción lingüística. En muchos sitios webs especializados, mediante ejercicios en línea pueden mejorar la destreza de la escucha, para lo cual reciben realimentación inmediata y pueden volver a realizar el ejercicio las veces necesarias. Además, pueden efectuar otro tipo de ejercicios por medio de videos y sesiones en línea, prácticas de pronunciación y de gramática, con la ventaja de hacerlo en su propio tiempo y a su propio ritmo.

$\mathrm{Al}$ proporcionar a los docentes de la Escuela un programa de formación docente continua y en especial la integración de las TIC en la enseñanza de idiomas, podrían utilizarlas de manera más propicia y eficiente y, al mismo tiempo, facilitar a los estudiantes estrategias para sacarles el mejor provecho dentro del aula y fuera de esta, con el fin de mejorar sus destrezas comunicativas y su desempeño en el idioma meta.

\section{Objetivos de este estudio}

Este estudio tiene como objetivo general proponer una oferta de formación continua en la integración de las TIC en la enseñanza de idiomas, dirigida al profesorado de la Escuela de Lenguas Modernas. Para ello, se plantean los siguientes objetivos específicos:
1. Determinar el grado de alfabetización digital del profesorado de la Escuela de Lenguas Modernas, con el propósito de conocer las necesidades de capacitación en este nivel.

2. Identificar necesidades del profesorado con respecto a una eficaz integración de las tecnologías digitales en la adquisición de lenguas extranjeras.

\section{Metodología y resultados}

La idea de realizar esta investigación surgió como consecuencia de otro estudio efectuado en conjunto con tres investigadoras del Programa de Tecnologías Avanzadas (PROTEA) de la Facultad de Educación. Se percibió la necesidad de formación que tienen los docentes universitarios con respecto a la integración de las TIC, en especial de un sustento teórico para lograrla de la manera más acertada. En ese momento, se acordó efectuar esta investigación, recolectar insumos de los docentes de ambas unidades académicas y, con base en estos, elaborar una propuesta para un programa de formación continua más acorde con las necesidades reales.

Con este fin, en Asamblea de Escuela de julio de 2013, se pasó un cuestionario de opinión a los sesenta y un profesores presentes ese día. Este cuestionario contenía cinco secciones con diecisiete preguntas sobre información general de tipo socioprofesional, acceso y uso de las TIC, formación en su uso, experiencias con las TIC en actividades de clase y extraclase y, finalmente, expectativas y necesidades de formación del profesorado. Las respuestas a esas preguntas permitirían conocer el grado de alfabetización digital de los docentes, así como sus experiencias, 
intereses y necesidades de recibir capacitación.

Además del cuestionario a docentes, se llevó a cabo un grupo focal con veinte profesores (coordinadores o directores de proyectos o de sección), con la finalidad de profundizar y validar los hallazgos preliminares obtenidos con el cuestionario. Se buscaba que tuvieran un diálogo con mayor profundidad entre ellos y compartieran ideas, experiencias y patrones, en aras de llegar a conclusiones más reales.

Por otro lado, por ser los estudiantes los actores principales y la razón de ser de la docencia, su participación se consideró clave para tener información más completa al estructurar un programa de formación continua. Con este fin, se pasó una encuesta a una muestra de doscientos estudiantes, la cual debieron llenar en una de sus clases. Este instrumento cuenta con dieciséis preguntas sobre el uso que ellos hacen de las TIC en su vida personal y académica.

\subsection{Discusión y resultados del cues- tionario a docentes de la Escuela de Lenguas Modernas}

De acuerdo con los datos obtenidos en la encuesta, el perfil de los docentes de la Escuela de Lenguas Modernas se podría definir con las siguientes características: el $77 \%$ de los participantes son personas entre 26 y 50 años, y un $23 \%$ se ubica entre 51 y 60 años de edad; el $70 \%$ son mujeres y el $28 \%$ hombres, mientras un participante no indica su sexo. De ellos, el 46\% están nombrados como interinos y el 51\% en propiedad; dos no contestaron.

En cuanto a sus títulos profesionales, el $62 \%$ tiene un bachillerato uni- versitario, el 26\% obtuvo además una licenciatura, el 80\% posee (además del bachillerato o licenciatura) una maestría, el 11\% cuenta con un doctorado y un $7 \%$ ha realizado otros estudios en diferentes campos.

En relación con su jornada de trabajo, el 20\% trabaja para la Universidad de Costa Rica por medio tiempo o menos, el $15 \%$ lo hace por tres cuartos de tiempo y el 65\% con una jornada de tiempo completo o más. El 69\% de ellos trabajan exclusivamente para esta Universidad, mientras el 30\% ejercen cargos en otras instituciones. El 34\% tiene diez años o menos de experiencia docente, el 56\% entre 11 y 20 años y el 10\% más de 25 años.

De estos datos, se puede deducir que cerca de una cuarta parte del personal docente creció y ha enseñado la mayor parte de su vida sin dispositivos tecnológicos, razón por la cual posiblemente no vean el potencial de las TIC en la docencia. Además, un 67\% imparte cursos de bachillerato universitario, cuyo enfoque está centrado, en la mayoría de los cursos, en el desarrollo de las cuatro macrodestrezas comunicativas: escucha, habla, lectura y escritura.

El resto de los participantes, el 33\%, trabaja también en el nivel de licenciatura, posgrado o venta de servicios y en cursos de otras carreras, los cuales están más dirigidos a otro tipo de contenidos, como por ejemplo literatura, enseñanza de idiomas y venta de servicios.

Esta información revela la importancia de un programa de formación continua apropiado, pues la mayoría de los docentes trabaja en cursos de desarrollo lingüístico y de destrezas comunicativas que los estudiantes podrían mejorar de manera autónoma con las herramientas adecuadas. 
En el cuestionario también se les preguntó acerca de los recursos informáticos que utilizan en su vida personal. Los más usados por los docentes en sus actividades personales son el correo electrónico (90\%), internet (87\%), programas de ofimática $(66 \%)$ y páginas web (52\%); es decir, los utilizan comúnmente como herramientas de comunicación y en un nivel básico. También se les preguntó sobre el uso en su labor docente, con el fin de corroborar si conocían otras herramientas y programas que los empoderarían tanto a ellos como a sus estudiantes en el proceso de enseñanza-aprendizaje. El gráfico 1 contiene los recursos y programas informáticos que usan en sus clases.

\section{Gráfico 1 \\ Uso de recursos y programas informáticos en la clase}

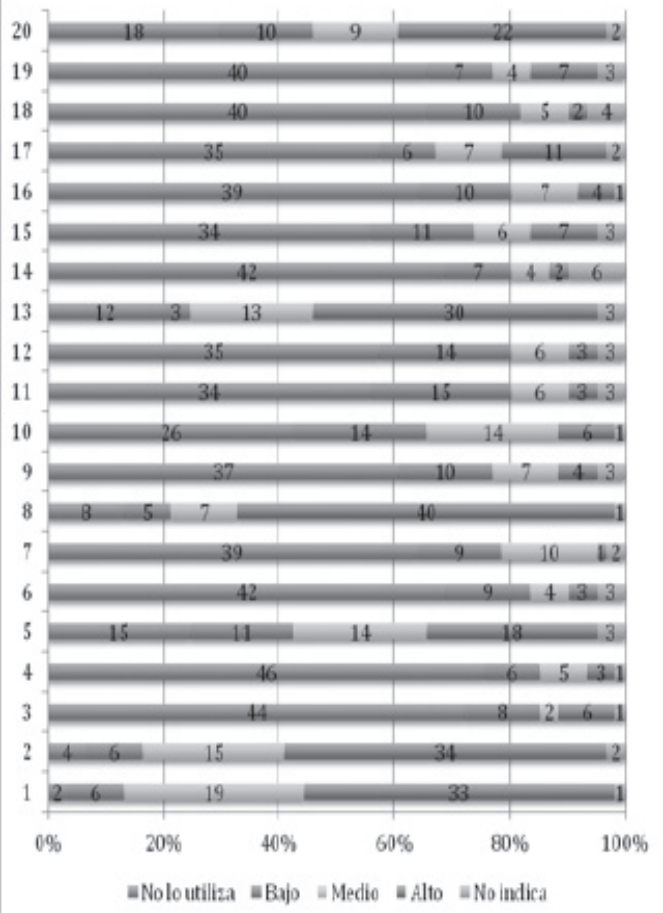

De la información anterior se deduce que los participantes usan los recursos informáticos en su vida personal de manera similar a como lo hacen en sus clases; el correo electrónico es el más utilizado con sus estudiantes (66\%), seguido por internet $(56 \%)$, presentaciones en multimedia (54\%) y diferentes programas de ofimática (49\%). Por el contrario, los recursos menos empleados son los chats (5\%), editores de audio y video (11\%), wikis (18\%) y blogs (3\%), los cuales precisamente permitirían a los estudiantes interactuar con otros y aprender de manera colaborativa con sus pares o con hablantes nativos del idioma meta.

Según se desprende del análisis del cuestionario, los docentes usa las herramientas que parecen ser más accesibles, y no necesariamente las que fomentarían en los alumnos destrezas para construir su propio conocimiento. Una posible razón podría ser que pocos han tenido la oportunidad de capacitarse en cómo hacer una efectiva integración de las TIC desde el punto de vista pedagógico. Muchos las emplean de manera intuitiva y con un poco de capacitación obtenida por ellos mismos en academias o por medio de amigos.

Otro aspecto relevante para explicar cuánto y cómo usa los diferentes recursos este grupo de docentes, está relacionado con la plataforma informática que está utilizando. La gran mayoría de ellos (40\%) solo usan Microsoft, por lo cual relacionan estos recursos más con la ofimática y búsquedas en Internet que con otros programas más específicos para el aprendizaje o la mejora de las destrezas comunicativas. Esto podría deberse a que en nuestro país ha habido una tendencia grande a ser consumidores masivos de las herramientas de Microsoft. 
La docencia es un área en donde también se ha mantenido esa tendencia, y no es sino hasta el año 2013 cuando la Universidad de Costa Rica ha migrado al software libre. Esto probablemente hará que los docentes aprendan a utilizar una mayor cantidad de programas de uso libre en los próximos años y, de esa forma, conocer más herramientas aplicables en la enseñanza, aunque no necesariamente en la integración de las TIC de manera intencionada para mejorar el desempeño de los estudiantes.

Como este estudio se propone detectar las necesidades de formación, en el cuestionario se incluyó una pregunta para saber si los profesores están interesados en capacitarse y en qué aspectos. El siguiente gráfico resume las razones seleccionadas de una lista de ocho afirmaciones.

\section{Gráfico 2}

Razones para participar en cursos de formación en el uso de las TIC

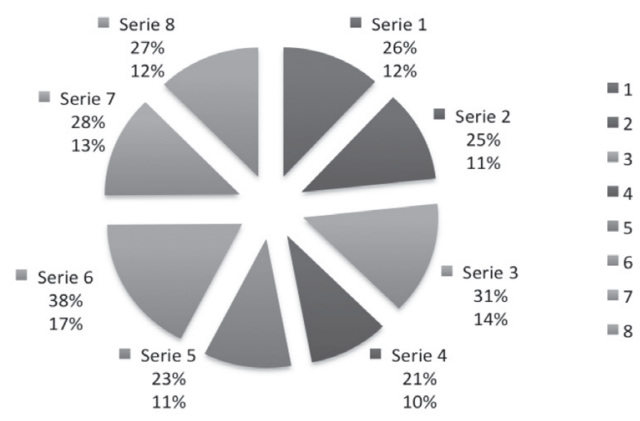

1- Necesito mejorar mi desempeño en el nivel técnico.

2- Necesito conocer mejor los programas disponibles en el mercado.

3 - Me son muy útiles en mi desempeño personal.

4- Me siento muy cómodo(a) con estas tecnologías y me gusta trabajar con ellas.

5- Son indispensables en mi desempeño profesional.
Pocos docentes (10\%) manifiestan interés en capacitarse en los aspectos teóricos para lograr una integración adecuada de las TIC en la enseñanza. Esto quizá se deba a que muchos de ellos no son graduados del área de la pedagogía sino de otras especialidades, por lo cual no les parece importante el componente pedagógico ni el sustento teórico. Por el contrario, algunos sí están pensando en aprender a usar otro tipo de herramientas colaborativas, como chats (10\%), mapas conceptuales $(11 \%)$, software especializado (7\%) y plataformas bimodales (15\%), que permitirían a los estudiantes mejorar su desempeño. Aprendiendo a sacar mayor provecho a Internet, por medio de redes sociales especializadas, los estudiantes podrían compartir y mejorar destrezas comunicativas, no solo con sus pares sino también con hablantes nativos y estudiantes de otras universidades con quienes pueden discutir y realizar proyectos en común.

Un aspecto digno de resaltar es el hecho de que si bien pocos aseveraron usar herramientas colaborativas en sus clases, quienes las utilizan (durante la clase y extraclase) lo hacen con frecuencia. Con el fin de favorecer el desempeño en las diferentes destrezas comunicativas, los participantes utilizan por ejemplo blogs y wikis, en especial para los cursos de composición, pues les sirven como un medio para analizar y comentar ejemplos de composiciones y poder mejorarlas. Para aumentar las destrezas orales y de escucha, por otro lado, recurren a videos y foros de discusión, en donde los estudiantes pueden participar desde sus casas. Con el propósito de mejorar la comprensión de lectura, se les asignan lecturas relacionadas con los 
temas discutidos en clase, en algunos sitios específicos de Internet, como se observa en el cuadro 1.

Los docentes participantes mostraron mucho interés en recibir cursos de formación continua en el área de integración de las TIC y también especificaron sus principales necesidades y expectativas. Algunos mencionaron la importancia de aprender a utilizarlas en el contexto educativo y no solo como instrumentos de ofimática que muchos mencionaron. Además, piden a la Escuela tener una política al respecto y ofrecerles oportunidades de capacitación en los tiempos apropiados para ellos y en la misma unidad académica, como lo muestra el cuadro 2.

\section{Cuadro 1 \\ Actividades de clase y extraclase en las que los docentes emplean TIC}

\begin{tabular}{lcc}
\hline \multicolumn{1}{c}{ Tipo de actividad } & En la clase & Extraclase \\
\hline Complementar la oralidad del idioma con escucha y ejercicios de re- & $*$ \\
petición y comprensión & $*$ \\
Ampliar aspectos de historia y civilización con videos cortos e intere- & \\
santes que luego son discutidos & $*$ \\
Presentaciones de diapositivas & $*$ \\
Foros (blogs) de discusión & $*$ \\
Videos: videoconferencias por medio de Google; práctica de traduc- \\
ción simultánea, consecutiva con videos seleccionados en la red; rea- \\
lizar videos para calificar la parte oral (Windows, Movie Maker) \\
Mapas conceptuales \\
Traducción de documentos \\
Uso de software para el proceso de traducción y subtitulado \\
Foros de discusión acerca de los temas vistos en clase \\
Word y Excel para formatos de documentos \\
Uso de escritos virtuales animados por medio de Open GL para pre- \\
sentaciones de multimedios, ofimática y audio al mismo tiempo \\
Quizzes y ejercicios en línea \\
Wikis para analizar writing samples \\
Búsqueda de información acerca de un tema específico para trabajos \\
en grupo \\
Referencias a guías para formatos de documentación \\
Pizarras: electrónicas, interactivas, escritorios virtuales \\
Diferentes sitios con los cuales el alumno puede ampliar, de forma \\
más libre y amena, puntos vistos en clase \\
$\begin{array}{l}\text { Intercambio de ideas con jóvenes de otros países y cultura en el idio- } \\
\text { ma que se está aprendiendo, lo cual enriquece mucho } \\
\text { Correo electrónico: Peer review, corrección de errores }\end{array}$ \\
\end{tabular}


Blogs: Trabajo para realizar en blogs visitados antes por el profesor para verificar seriedad; blogs de discusión: los estudiantes deben practicar vocabulario, gramática, escucha y escritura por medio de wikis y blogs

Internet: Búsqueda de información disponible de fuentes confiables, textos comparables y paralelos

Websearches

Wikispace: en los cursos de composición, wikis para analizar writing samples

Traducción de documentos

Búsqueda de información

Uso de software

Búsqueda y organización de información

Los estudiantes deben trabajar prácticas en línea desde sus casas como material complementario

Dejo actividades interactivas disponibles en mi página web

Web quests

Diseño de un portal de materiales en Google Drive

Realizar consultas vía chat; los estudiantes graban sus tareas en audio y video

\section{Cuadro 2 \\ Principales necesidades y expectativas de capacitación de los docentes para el uso de las TIC en el trabajo}

\begin{tabular}{|c|c|}
\hline Necesidades & Expectativas \\
\hline $\begin{array}{l}\text { Mejor conectividad de la red, es decir, mejora } \\
\text { en el laboratorio, mayor rapidez }\end{array}$ & $\begin{array}{l}\text { Mayor capacidad para los profesores y quitar- } \\
\text { les el miedo al uso de la tecnología }\end{array}$ \\
\hline Capacitación & $\begin{array}{l}\text { Aprender a utilizar las herramientas en función } \\
\text { de las clases }\end{array}$ \\
\hline Acceso a equipo & $\begin{array}{l}\text { Tener el equipo necesario para la aplicación de } \\
\text { las TIC }\end{array}$ \\
\hline \multicolumn{2}{|l|}{ Diseñar más cursos en línea } \\
\hline Uso de Prezi & Usarlo efectivamente \\
\hline \multicolumn{2}{|l|}{ Capacitación en tecnologías de traducción } \\
\hline \multicolumn{2}{|l|}{ Inversión en licencias } \\
\hline Cómo sacar mayor provecho de los programas & $\begin{array}{l}\text { Cursos que capaciten para ello y sean persona- } \\
\text { lizados. }\end{array}$ \\
\hline $\begin{array}{l}\text { Romper el paradigma de dependencia de } s o f t \text { - } \\
\text { ware de Microsoft }\end{array}$ & $\begin{array}{l}\text { Que se enseñe a docentes y estudiantes que las } \\
\text { TIC son diferentes de Windows y MS Office. }\end{array}$ \\
\hline
\end{tabular}


Necesito mejorar mi desempeño en las TIC

Tengo la necesidad de encontrar una plataforma en línea que permita a mis estudiantes desarrollar la escritura de manera colaborativa (escribir, editar, mejorar, etc.)

Para los cursos que imparto, me gustaría saber de edición de videos

Plataformas bimodales

Aprender a usar mejor Moodle

Software para obtener archivos de audio y video Manejo de blogs

Información sobre software libre

Integración del blogs más funcional; también conocer más herramientas

Ampliar conocimientos

Mejor información del potencial existente

Dar a los alumnos más recursos en línea y colaborar conjuntamente en proyectos con hablantes nativos

El problema es que, sin importar la herramienta, el centro de recursos no le da el mantenimiento a los laboratorios de cómputo, y eso desmotiva mucho.

Aprender sobre hojas electrónicas y el sistema Office

Actualizar el equipo de cómputo de las oficinas

Actualizaciones frecuentes

Poder trabajar con las TIC en un nivel avanzado

Aplicaciones para mejorar la enseñanza

Capacitación en edición de audio y video, al igual que de páginas webs

La capacitación en estas herramientas es muy importante siempre y cuando se tome en cuenta que algunos no somos muy diestros y necesitamos ir despacio.

Aprender a manejar las diferentes herramientas
Que las TIC en realidad se usaran para empoderar y liberar, en lugar de perpetuar modelos de dependencia creada.

Quisiera lograr un mayor dominio en el empleo de las TIC.

Algunos profesores están usando ciertas herramientas pero no comparten sus experiencias o tan solo lo hacen con pocos colegas. Me gustaría que los profesores compartieran cómo están usando las herramientas.

Llevar un curso y tener un nivel alto en esta área para poder enseñarle a los estudiantes

Incrementar mi conocimiento sobre ellos

Poder crear páginas de apoyo a mis cursos

Tener software libre

Recibir información

Poder hacer mis clases más atractivas e interesantes para alumnos y facilitar la labor docente Agilizar procesos

Capacitación dentro de la Escuela de Lenguas Modernas

Talleres de capacitación en verano

Lograr un desempeño apto y utilizar al máximo aplicaciones disponibles

Por lo menos una vez al año

Formaciones dirigidas a la enseñanza

Recibir cursos en donde paso a paso podamos elaborar un producto, p. ej. una página web

Que los recursos funcionen, pues de nada sirven las capacitaciones si muchas veces los equipos aquí no funcionan 
Uso de la plataforma de la UCR

El acceso a internet wi-fi debe ser automático

Uso de software libre, aprender a utilizar Prezi, uso de Microsoft Excel, uso correcto de las redes sociales para las clases

Conocer de nuevos recursos en línea (programas, páginas gratuitas)

Cómo captar documentos sonoros

Que los docentes entiendan que deben capacitarse en el uso de las TIC

Romper con la monocultura tecnológica

Uso de software libre, uso avanzado de plataforma de la UCR, cómo integrar las tecnologías al currículo y a los cursos existentes
Poder practicar, que no sea solo un curso teórico sino en un laboratorio y poder incluso diseñar un curso

Poder hacerlo por mí misma y no depender del centro de recursos; llevar el curso en Didáctica Universitaria.

Que Lenguas Modernas obligue a los docentes a capacitarse y que se ofrezca variedad de capacitaciones todo el tiempo.

Capacitaciones que empoderen a los docentes para usar tecnologías y comprender el papel de estas en la educación.

\subsection{Resultados del grupo focal con docentes de Lenguas Modernas}

Con el fin de triangular la información obtenida con el primer instrumento (61 profesores en Asamblea de Escuela), en junio de 2013 se realizó un grupo focal con 20 docentes, quienes además eran coordinadores de diferentes programas y proyectos de la Escuela de Lenguas Modernas.

La metodología utilizada en este grupo focal fue la siguiente: primero se dividió a los participantes en cinco grupos, en los cuales debían discutir once preguntas y, de manera consensuada, debían anotar una sola respuesta para cada una de ellas.

Sin embargo, en esta investigación, y con base en la discusión generada por los participantes del grupo focal, los sujetos de estudio tienen principalmente dos propósitos en mente: las usan como herramientas para la evaluación de los aprendizajes (por ejemplo, tecnologías de audio y video para grabar a los estudiantes haciendo diferentes prácticas) y como herramientas para el estudio individual (asignando prácticas en línea, algunas creadas por ellos o de algún sitio web, que estimulan la escucha, la comprensión de lectura, la pronunciación, la escritura e incluso la gramática). Solo en dos grupos se mencionó el uso de blogs y wikis como herramientas para la interacción social, y en la mayoría el uso del chat.

En cuanto al uso de dispositivos portátiles con acceso a Internet para la búsqueda de información y para fomentar el aprendizaje ubicuo, es importante señalar que esta pregunta generó más discusión en los diferentes grupos y tuvo menos respuestas concretas. Los observadores anotaron como punto importante que quienes no 
asignaban tareas o actividades en línea afirmaban no hacerlo por creer que los estudiantes no tenían suficientes equipos ni acceso a Internet. Posiblemente, otra razón para esta controversia esté relacionada con la falta de capacitación sobre cómo integrar las TIC desde una perspectiva pedagógica.

En relación con el acceso de los profesores al equipo de manera suficiente para lograr los objetivos de la clase y la adquisición del idioma meta por parte de los estudiantes, los grupos de discusión coinciden en que éste es poco. Se mencionan factores como el hecho de usar a veces los laboratorios como aulas (precisamente por carecer la Escuela de suficientes aulas); además, los equipos de los laboratorios se usan muchas horas al día y algunas veces no tienen mantenimiento continuo; respecto a los programas disponibles y los equipos de apoyo (los cuales incluyen, además de la laptop, un proyector, parlantes y extensiones) requieren de actualización y mantenimiento constante, el cual no se da de manera oportuna. Por otro lado, consideran que en las oficinas hay poco equipo, a veces solo una computadora para muchos docentes.

Además, señalan tener problemas con la conexión a Internet, la cual es mejor en algunos laboratorios que en otros. En las aulas es muy difícil conectarse, y cuando se logra es difícil mantener la conexión estable. Por esta razón, los trabajos extraclase no se pueden asignar desde las oficinas; los docentes deben hacerlo desde sus propias casas (si tienen conexión a Internet) o buscar otro lugar. Uno de los grupos mencionó también como deficiencia la falta de capacitación de los docentes.

Otra de las preguntas del grupo focal se refería al tipo de equipos y pro- gramas informáticos que más utilizan en la clase y extraclase. La laptop, el proyector, la grabadora y los clickers son los más citados, además de grabadoras periodísticas y kindles. En los programas, destacan las herramientas de Microsoft, videos de YouTube y sitios web para prácticas en línea. En la enseñanza del francés, también cuentan con una plataforma especial con material sonoro y ejercicios para desarrollar las diferentes destrezas comunicativas.

Con respecto al dominio de la tecnología por parte de docentes y estudiantes, la mayoría de los participantes de este grupo focal piensa que los estudiantes tienen un conocimiento más avanzado y actualizado pero lo utilizan más como una herramienta social y no como herramienta de aprendizaje; para ellos, los docentes, en cambio, tienen menos conocimiento y requieren más capacitación. Asimismo, mencionan su interés por conocer aplicaciones más prácticas y vencer el miedo a la tecnología.

Por otro lado, en cuanto a las limitaciones para integrar las TIC tanto en la Universidad en general como en la Escuela en particular, los grupos apuntan como un factor muy importante la falta de tiempo, pues se requiere diseñar, mantener, actualizar y revisar trabajos en línea. Además, la evaluación de los cursos no contempla este tipo de actividades, por lo cual no se puede obligar al estudiando a hacerlas, aun cuando se haya invertido mucho tiempo. Otro factor relevante en el cual coinciden los grupos es la falta de capacitación y divulgación de talleres y cursos de este tipo, y piden a la Escuela involucrarse más en este aspecto. Uno de los grupos también incluye el factor cultural (resistencia al cambio) y 
el alto costo de las licencias de programas especializados.

Con el fin de superar esas limitaciones, los participantes sugieren tener una mayor cantidad de laboratorios, con horarios especiales, abiertos también al estudiantado, así como una mayor cantidad de equipos de buena calidad en constante mantenimiento y con programas especializados, tanto en las oficinas como en los laboratorios. En este sentido, recomiendan dedicar un mayor presupuesto para adquirir mejor y mayor cantidad de equipo y para mejorar la infraestructura y la conexión a Internet. Destacan como un punto muy importante capacitar a los docentes y que exista un mayor compromiso de la Escuela con los entes involucrados en la capacitación, con el fin de que, cuando creen aulas virtuales durante un curso, por ejemplo, se mantengan por más tiempo y no las cierren.

Cabe señalar que ninguno de los grupos mencionó nada sobre actualizar los contenidos de los programas disponibles en la actualidad ni tampoco sobre realizar un proceso de sensibilización, acompañamiento y motivación para superar la barrera del cambio y uso de las TIC. Esto nos lleva a reflexionar sobre la importancia de efectuar cambios en la cultura de aprendizaje, mediación y flexibilidad que el uso de la tecnología demanda. Así como la tecnología cambia en tiempos muy cortos, cambiará no solo la forma de la información sino también las destrezas y estrategias que deben desarrollar los aprendices de idiomas extranjeros. Por ello, los docentes deben adaptarse y actualizarle de manera continua.

Pese a las limitaciones de tiempo para capacitarse, existe gran interés de parte del profesorado por mante- nerse actualizado y aprovechar las TIC en la docencia. Según plantea la mayoría, un proceso de capacitación debe ser bimodal, para poder interaccionar con los instructores y, a la vez, contar con tiempo más flexible. Además, esta modalidad involucra tanto la parte humana (presencial) como la tecnológica. En torno a la disponibilidad horaria, la mayoría coincide en la bimodalidad a principio de cada semestre (pues hay pocas actividades durante este tiempo), un horario flexible en las clases presenciales y poder comunicarse fácilmente con los encargados de la capacitación por si surgiera alguna duda.

Al concluir el grupo focal, los participantes expresaron que, para que un programa de educación continua en materia de integración de TIC sea de su interés y provecho, debe haber una relación entre la pedagogía y las TIC, por cuanto su uso debe tener sentido y ser práctico y aplicable ciento por ciento a la labor docente. Esta información revela que los participantes en este grupo focal son más conscientes de la importancia de la pedagogía y su relación con el uso de las TIC en la docencia; tal vez por ser profesores con más años de experiencia y haber ocupado cargos de coordinadores de sección, han lidiado con problemas de currículo, de evaluación de los aprendizajes y de temas más pedagógicos.

\subsection{Resultados del cuestionario apli- cado a estudiantes de la Escuela de Lenguas Modernas}

Los estudiantes son la razón de ser de todo centro educativo, por lo cual la formación continua de los docentes debe estar íntimamente relacionada con las 
necesidades de los educandos y responder a ellas. Con este fin en mente, se creó un cuestionario para conocer sus percepciones sobre el uso de la tecnología y cómo esta impacta su aprendizaje.

Se escogió una muestra al azar de 207 estudiantes entre diferentes grupos de las carreras de Enseñanza del Inglés y Enseñanza del Francés. De ellos, 147 son mujeres y 60 hombres. En su gran mayoría son personas muy jóvenes: el 98\% son menores de 35 años y solo un $2 \%$ tiene entre 41 y 45 años de edad. De este grupo, el 100\% vive en San José $(70 \%)$ o en el resto de la
Gran Área Metropolitana (30\%). No se encontró ninguno de las zonas de Guanacaste, Puntarenas o Limón. El 92\% son estudiantes de entre primer y tercer año (de cualquiera de las dos carreras), y un 8\% está en su último año de bachillerato.

La gran mayoría de los participantes posee y usa varios dispositivos tecnológicos: el 85,5\% reportó tener teléfono celular, el 79\% una computadora portátil, el $46 \%$ una computadora de escritorio y el 40,5\% una tableta. Estos dispositivos los usan casi todos los días y como mínimo dos o tres veces por semana.

\section{Cuadro 3}

Frecuencia y formas en que los estudiantes usan los dispositivos tecnológicos

\begin{tabular}{lcccc}
\hline \multicolumn{5}{c}{ Formas en las cuales los estudiantes utilizan los dispositivos } \\
\hline \multicolumn{1}{c}{ Frecuencia } & $\begin{array}{c}\text { En su vida } \\
\text { diaria }\end{array}$ & $\begin{array}{c}\text { Durante las } \\
\text { clases en la } \\
\text { Escuela de Len- } \\
\text { guas Modernas }\end{array}$ & $\begin{array}{c}\text { Para } \\
\text { reforzar lo } \\
\text { aprendido }\end{array}$ & $\begin{array}{c}\text { Como apoyo para } \\
\text { realizar trabajos } \\
\text { extraclase }\end{array}$ \\
Casi todos los días & 97,58 & 36,71 & 39,61 & 45,37 \\
Dos o tres veces /semana & 2,42 & 36,23 & 34,29 & 37,48 \\
Una vez / semana & - & 4,84 & 8,21 & 10,14 \\
Rara vez & - & 17,40 & 15,45 & 6,73 \\
Nunca & - & 4,35 & 2,42 & 0,44 \\
\hline
\end{tabular}

Con base en los datos anteriores, podemos concluir que los estudiantes utilizan los dispositivos tecnológicos no solo como medios para socializar sino también como herramientas de aprendizaje, dentro del aula y fuera de esta. Los emplean con mayor frecuencia en su vida diaria; sin embargo, el $73 \%$ también los usa a menudo en sus clases y para reforzar lo aprendido (un mínimo de dos o tres veces por semana), mientras cerca de la mitad los utiliza para realizar trabajos extraclase casi todos los días, y solamente menos del 1\% afirma no usarlos nunca.

De acuerdo con los datos del cuestionario, el 86\% de los estudiantes afirma tener un nivel de experticia intermedio o avanzado, y el 14\% un nivel básico. Cabe destacar que el 86,5\% de ellos ha recibido cursos en línea (de diferentes tipos, no para el aprendizaje del inglés) y el 13,5\% nunca ha tenido esta experiencia. Además, el 98,5\% afirma tener acceso a Internet en sus casas de habitación, el 87,4\% en la Universidad, un 
Cuadro 4

Recursos multimedia que usan los estudiantes

\begin{tabular}{lc}
\hline \multicolumn{1}{c}{ Recursos multimedia } & $\begin{array}{c}\text { Porcentaje de estudiantes que em- } \\
\text { plean estos recursos multimedia }\end{array}$ \\
\hline Procesador de texto & $100 \%$ \\
Hoja de cálculo & $76,81 \%$ \\
Base de datos & $27,53 \%$ \\
Presentaciones temáticas & $99,03 \%$ \\
Programas de dibujo & $90,82 \%$ \\
.gif & $31,88 \%$ \\
.jpeg & $43 \%$ \\
.jpg & $82,12 \%$ \\
.png & $22,22 \%$ \\
Mapa de bits (.bmp) & $9,66 \%$ \\
Otro & $4,83 \%$ \\
Editores de video & $65,21 \%$ \\
Navegadores de Internet & $100 \%$ \\
Curso en línea & $20,28 \%$ \\
Correo electrónico & $99,51 \%$ \\
Redes sociales & $97,58 \%$ \\
Wikis & $66,67 \%$ \\
Foros & $53,14 \%$ \\
Chats & $67,63 \%$ \\
Blogs & $57,97 \%$ \\
Videos en línea & $98,55 \%$ \\
Buscadores & $100 \%$ \\
Google & $100 \%$ \\
Altavista & $0,44 \%$ \\
Yahoo & $6,28 \%$ \\
Metros dros webs específicos & $0 \%, 63 \%$ \\
\hline & $27,53 \%$ \\
Otras herramientas & $52,65 \%$ \\
\hline
\end{tabular}


$15,4 \%$ en un café Internet y el $11,5 \%$ en otros lugares, como por ejemplo en el trabajo o en sus celulares con Internet móvil. Ninguno de ellos se quejó de falta de acceso, lo cual llama mucho la atención, pues, en la información aportada por los docentes en el primer instrumento de este estudio, uno de sus temores era precisamente que los estudiantes no tuvieran acceso a la red.

Con el fin de corroborar el nivel de experticia de los estudiantes, se les preguntó cuáles recursos tecnológicos usan, en qué formato guardan imágenes y cuáles herramientas emplean para buscar información en Internet. Los resultados se consignan en el cuadro 4.

Como se observa en el cuadro, los recursos más usados por los estudiantes son el procesador de texto, Internet, redes sociales, videos en línea, presentaciones temáticas y programas de dibujo; y los menos usados son los cursos en línea y las bases de datos, quizá porque estos dos recursos se usan solo con fines muy específicos y son más técnicos. Los otros recursos, como blogs, wikis, foros y chats (es decir, aquellos que fomentan aprender de manera colaborativa), los usan con alguna frecuencia como herramientas de comunicación y no para el aprendizaje; probablemente los profesores deberían fomentar más su uso.

De este cuadro también se infiere que los participantes están muy identificados con el buscador de Google, por cuanto todos lo mencionaron; además, más de la mitad utiliza Google académico y otros sitios webs más específicos, pues desean información más especializada y confiable, lo cual demuestra un mayor grado de criticidad en cuanto a la información que circula en Internet. Solo cinco estudiantes ci- taron otros buscadores, como Internet Explorer y Bing.

Las respuestas al último punto de este instrumento fueron muy reveladoras: a excepción de un estudiante, todos los demás respondieron afirmativamente ante la pregunta de si consideraban que los recursos tecnológicos podían ayudarlos a mejorar su desempeño en el idioma meta. Este resultado debe hacernos reflexionar acerca de la importancia asignada al papel de las TIC para el aprendizaje por parte de los estudiantes y los docentes. Asimismo, es preciso analizar la posición y el compromiso que la Escuela debería tener al respecto porque, según lo manifiestan ambas poblaciones, los recursos tecnológicos deberían ser más accesibles para todos.

\section{Conclusiones y recomendaciones}

De la información aportada por las fuentes consultadas, se puede concluir que tanto los docentes como los alumnos de la Escuela de Lenguas Modernas utilizan de muy diferentes formas los recursos tecnológicos a los cuales tenemos acceso en la actualidad.

Por un lado, de las respuestas al cuestionario concluimos que el personal docente las está integrando, aunque de manera tímida, pues muchos no han recibido la capacitación necesaria para sacarles el mejor provecho desde el punto de vista pedagógico. Lo que han hecho es trasladar a la enseñanza, de modo intuitivo, el conocimiento adquirido de manera personal.

Del grupo focal se concluye que los docentes básicamente usan las TIC de dos formas: como herramientas de evaluación y para el estudio individual del 
estudiante, pero no las utilizan para la interacción social ni para facilitar el aprendizaje ubicuo, dos usos importantes encontrados en el estudio de Frank (2008) y ausentes en la presente investigación. Además, debido a la restricción de tiempo, sugieren realizar cursos de capacitación de manera bimodal, con los encuentros presenciales durante los meses de vacaciones y en horas de la mañana, para poder participar con más flexibilidad.

Otro punto importante es contar con un certificado al finalizar la capacitación, como reconocimiento al esfuerzo y tiempo dedicado, de manera que se sientan aún más estimulados para así apropiarse de las tecnológicas en general, tanto para su vida personal como para mejorar su desempeño en el aprendizaje de idiomas. Todos ellos poseen algún tipo de dispositivo, en especial móviles con acceso a Internet. Este dato es relevante para que la Escuela tome decisiones acertadas sobre la integración de las TIC en el aprendizaje de idiomas, tal y como se ha recomendado para la reacreditación de los bachilleratos de Inglés y de Francés.

De acuerdo con los comentarios de los estudiantes, el nivel de equipamiento y de acceso a Internet es mucho mayor que el mencionado por los docentes, pues, como casi todos tienen dispositivos móviles, no dependen de que la Escuela o la Universidad se lo provea o les dé acceso a la red. A pesar de ese alto nivel de equipamiento conectado a la red y del conocimiento de varios recursos informáticos, especialmente de parte de los alumnos, hasta ahora no se realiza una verdadera integración ni se fomenta el aprendizaje ubicuo. Esto probablemente esté rela- cionado también con una óptima capacitación de los docentes, en especial en el área pedagógica relacionada con una efectiva integración de las TIC en la docencia (Cope y Kalantzis, 2009) y, en el caso particular de la Escuela, en el aprendizaje de idiomas.

Las conclusiones de este estudio destacan la necesidad del profesorado de Lenguas Modernas de contar a corto plazo con un programa de desarrollo profesional, en el cual se incluyan talleres y cursos en diferentes modalidades, para promover la integración pedagógica de las TIC. Este programa debe basarse en resultados de investigaciones y experiencias anteriores, y también tomar en cuenta recomendaciones emitidas por especialistas en pedagogía y en la enseñanza de idiomas, y además por la misma población docente de la Escuela.

Tres investigadoras de la Facultad de Educación, por medio del Programa de Tecnologías Avanzadas (PROTEA) y del Instituto de Investigación en Educación (INIE), y esta autora han estado trabajando de manera simultánea en esta investigación sobre el estado actual de la integración de las TIC por parte del profesorado de las dos unidades académicas, Educación y Lenguas Modernas. Los resultados sirvieron de base para que las investigadoras del PROTEA y del INIE realizaran el diseño de una propuesta para un programa de educación continua dirigido a capacitar a los docentes en la integración de las TIC con el propósito de que las utilicen con objetivos pedagógicos y de manera intencionada (Ríos, Salas y Zúñiga, 2014).

Por todo esto, se recomienda crear un equipo interdisciplinario con profesores de Lenguas Modernas, PROTEA, 
INIE y expertos en la integración de las TIC en la enseñanza de idiomas, con la finalidad de adaptar la propuesta a las necesidades tanto del personal docente como de los alumnos de la Escuela. También, se propone que en los próximos congresos y seminarios se dé más importancia al tema de las TIC y que se invite a expertos en el transcurso del año a impartir talleres y seminarios, con especial énfasis en el fundamento teórico que respalda la integración de tecnología en la docencia.

Por último, se sugiere a la Escuela establecer una política explícita sobre el uso de las TIC, mediante la cual se fomente la integración y formación adecuada de su personal. Retomando las palabras de los docentes, también se recomienda a todos los actores involucrados en el quehacer de la Escuela asumir un mayor compromiso y hacer realidad la meta de contar con la capacitación requerida, los equipos necesarios y un buen acceso a Internet.

\section{Bibliografía}

Arnold, N. (2007). Technologicalmediated learning 10 years later: emphasizing pedagogical or utilitarian applications? Foreign Language Annals, 40 (1), 161-181.

Beatty, K. (2003). Teaching and Researching Computer Assisted Language Learning. Pearson Education Limited.

Chapelle, C. (1998). Multimedia CALL: Lessons to be learned from research on instructional SLA. Language Learning and Technology, 2 (1), 21-59.

Chapelle, C. (2008). Tips for teaching with CALL: Practical Approaches to Computer- Assisted Language Learning. Pearson Longman.

Cope, B. y Kalantzis, M., editores (2009). Ubiquitous Learning. Exploring the anywhere/anytime possibilities for learning in the age of digital media. University of Illinois Press, 264 pp.

Egbert, J. (2005). CALL Essentials: Principles and Practice in CALL Classrooms. Teachers of English to Speakers of other Languages, Inc. (TESOL).

Ellis, R. (1997). SLA Research and Language Teaching. Oxford: University Press.

Frank, V. et al. (2008). Optimal foreign language learning: the role of technology. College Park, MD: Center for Advanced Study of Language at the University of Maryland.

Hanson-Smith, E. y Rilling, S. (2006). Using Technology in Teaching Languages. Teachers of English to Speakers of other Languages, Inc. (TESOL).

Healy, J. M. (1998). Failure to connect: How computers affect our children's minds-for better and worse. New York: Simon \& Schuster.

Hubbard, P. (2005). A review of subject characteristics in CALL research. Computer assisted language learning, 18 (5), 351-368.

Krashen, S. (1981). Principles and practice in second language acquisition. English Language Teaching Series. London: Prentice-Hall International (UK) Ltd., 202 pp.

Levy, M. (1997). Computer-assisted language learning: Context and conceptualization. New York: Oxford University Press.

Lightbrown y Spada, N. (1999). How languages are learned. New York: Oxford University Press. 
Nunan, D. (1987). Methodological issues in research. Applying second language acquisition research. Adeleide, National Curriculum Resource Center: 143-71.

Pica, T. (1998). Second Language learning through interaction: multiple perspectives. In Regan, V. (ed.). Contemporary Approaches to Second Language Acquisition in Social Context. Dublin: University College Dublin Press: 9-31.

Ríos, K. (2013). Perfil de competencias docentes para el empleo de las TIC en cursos bimodales y virtuales en la Facultad de Educación de la Universidad de Costa Rica. Universidad TECVIRTUAL.

Ríos, K.; Salas, F. y Zúñiga, E. (2014). Informe Final Proyecto 305. Diagnóstico de necesidades de formación del profesorado de la Facultad de Educación de la UCR para la integración de las TIC en la docencia. Instituto de Investigación en Educación. Universidad de Costa Rica.

Salas, F. (2013). La integración de las TIC en la docencia en la UCR: es- tudio desde un enfoque sistémicocomplejo del personal docente que se certificó en el curso Educar para el futuro, versión universitaria en el período 2003-2006. Tesis doctoral, Programa de Doctorado Latinoamericano en Educación, Universidad de Costa Rica, San José, Costa Rica.

Swain, A. (1995). Principle and Practice in Applied Linguistics. Oxford University Press.

Yong, Z. (2003). Recent Developments in Technology and Language Learning: A Literature Review and Meta-analysis. Michigan State University. CALICO Journal, 21, (1), p. 7-27.

Warschauer, M. (1997). Computermediated collaborative learning: theory and practice. The Modern Learning Journal, 81 (iv), 470-81.

Vygotsky, L.S. (1962). Thought and Language. Cambridge, MA: MIT Press.

Vygotsky, L.S. (1978). Mind in Society. Cambridge, MA: MIT Press. 